\title{
Hybrid organic-inorganic materials for integrated optoelectronic devices
}

\author{
T. Wehlus ${ }^{*}, 1$, T. Koerner ${ }^{1}$, S. Nowy ${ }^{1}$, J. Frischeisen ${ }^{1}$, H. Karl ${ }^{1}$, B. Stritzker ${ }^{1}$, W. Bruetting ${ }^{1}$ \\ ${ }^{1}$ EPIV, University of Augsburg, 86135 Augsburg, Germany
}

Received XXXX, revised XXXX, accepted XXXX

Published online XXXX

PACS 01.30.-y, 33.57.+c, 85.70.Ge, 85.70.Sq, 42.65.-k

* Corresponding author: e-mail thomas.wehlus@physik.uni-augsburg.de, Phone: +49-821-598 3498, Fax: +49-821-598 3425

\begin{abstract}
Abstract

Abstract

Abstract

Abstract

Abstract

Abstract

Abstract

Abstract

Abstract

Abstract

Abstract
\end{abstract}

1 Introduction Organic electronics have been the subject of intensive research in the past years. Especially Organic light emitting diodes (OLED) have matured and are already used in organic electronic devices like displays or tv screens. OLEDs are incoherent, Lambertian light sources, they are ultrathin and independent of the surfaces structure as long as it is plane. By employing a semitransparent metal film into the OLED a microcavity is formed, which manifests in a modification of the emission properties and leads to a spatial and spectral redistribution of the emission of the microcavity OLED (see Fig. 1b). By choosing the right parameters the ratio between s- and p-polarized emitted waves can be adjusted [1].

Polarized light is essential for the use of non-linear optical devices based on the Faraday-effect [2,3]. A miniaturization of macroscopic magneto optical devices like an optical isolator or modulator, leading to an integration of one such device on a single chip, is desirable. Therefore a combination of magneto optical material and a polarized light source is beneficial. This makes microcavity OLEDs promising light sources for future generation integrated optoelectronic devices. Moreover waveguide modes, also called leaky modes, normally trapped in OLEDs should couple into a waveguide emedded under the OLED and thus reduce the fraction of light trapped inside the OLED.

The integration of such a microcavity OLEDs on a magneto optically active material with very high Faradayrotation is an interesting approach to modern optoelectronics. Garnet materials, like yttrium iron garnet (YIG) $\mathrm{Y}_{3} \mathrm{Fe}_{5} \mathrm{O}_{12}$, exhibit a high Faraday-Rotation and have low optical losses in the infrared region [4]. Bismuth iron garnet (BIG) $\mathrm{Bi}_{3} \mathrm{Fe}_{5} \mathrm{O}_{12}$ is the material with the highest known Faraday-rotation at room temperature. Therefore a combination of garnets and OLEDs seems to be promising.

Our ambition was to build a hybrid organic-inorganic modulator based on a BIG waveguide using a microcavity OLED as light source. In the last years we analyzed the formation of the BIG phase, the deposition of garnets on non garnet substrates and a wide range of substitutions to adjust the lattice constant to lay the foundations for a 
(a)

\begin{tabular}{|lr|}
\hline $100 \mathrm{~nm}$ & $\mathrm{Al}$ \\
\hline $30 \mathrm{~nm}$ & $\mathrm{Ca}$ \\
\hline $80 \mathrm{~nm}$ & $\mathrm{Alq}_{3}$ \\
\hline $80 \mathrm{~nm}$ & $\mathrm{TPD}$ \\
\hline $30 \mathrm{~nm}$ & PEDOT:PSS \\
\hline $125 \mathrm{~nm}$ & $\mathrm{ITO}$ \\
\hline & $\mathrm{SiO}_{2}$ \\
\hline
\end{tabular}

(b)

\begin{tabular}{|lr|}
\hline $100 \mathrm{~nm}$ & $\mathrm{Al}$ \\
\hline $30 \mathrm{~nm}$ & $\mathrm{Ca}$ \\
\hline $80 \mathrm{~nm}$ & $\mathrm{Alq}_{3}$ \\
\hline $80 \mathrm{~nm}-300 \mathrm{~nm}$ & $\mathrm{TPD}$ \\
\hline $1 \mathrm{~nm}$ & $\mathrm{~F}_{4} \mathrm{TCNQ}$ \\
\hline $15 \mathrm{~nm}-30 \mathrm{~nm}$ & $\mathrm{Ag}$ \\
\hline $30 \mathrm{~nm}$ & PEDOT:PSS \\
\hline $125 \mathrm{~nm}$ & $\mathrm{ITO}$ \\
\hline \multicolumn{3}{r}{$\mathrm{SiO}_{2}$} \\
\hline
\end{tabular}

(c)

\begin{tabular}{|lr|}
\hline $100 \mathrm{~nm}$ & $\mathrm{Al}$ \\
\hline $30 \mathrm{~nm}$ & $\mathrm{Ca}$ \\
\hline $80 \mathrm{~nm}$ & $\mathrm{Alq}_{3}$ \\
\hline $80 \mathrm{~nm}-300 \mathrm{~nm}$ & $\mathrm{TPD}$ \\
\hline $1 \mathrm{~nm}$ & $\mathrm{~F}_{4}$ TCNQ \\
\hline $15 \mathrm{~nm}-30 \mathrm{~nm}$ & $\mathrm{Ag}$ \\
\hline $30 \mathrm{~nm}$ & PEDOT:PSS \\
\hline \multicolumn{3}{|c|}{$\mathrm{nm}-125 \mathrm{~nm}$} & $\mathrm{ITO}$ \\
\hline $500 \mathrm{~nm}-1000 \mathrm{~nm}$ & $\mathrm{BIG}$ \\
\hline $30 \mathrm{~nm}$ & $\mathrm{YIG}$ \\
\hline & $\mathrm{SiO}$ \\
\hline
\end{tabular}

Figure 1 Various OLED stacks. (a) reference device with a transparent ITO anode on fused silica. (b) microcavity device with additional silver and $\mathrm{F}_{4} \mathrm{TCNQ}$ layer on ITO. (c) like stack (b) with an underlying BIG on YIG buffer. The ITO layer is optional.

magneto optical device based on BIG. We've already developed a buffer system to grow BIG on non-garnet substrates and analyzed the growth mechanisms of BIG films made by PLD on silicon, amorphous $\mathrm{SiO}_{2}$, gadolinium gallium garnet (GGG) $\mathrm{Gd}_{3} \mathrm{Ga}_{5} \mathrm{O}_{12}$ (001) and (111) substrates using insitu Reflection High Energy Electron Diffraction (RHEED), Transmission Electron Microscopy (TEM), Environmental Scanning Electron Microscopy (ESEM), Energy Dispersive X-ray Analysis (EDX), Atomic Force Microscopy (AFM), Rutherford Backscattering Spectroscopy (RBS), and X-ray Diffraction (XRD) measurements $[6,5$, 7,8].

For this study we startet with an optimization of the garnet material, with special focus on roughness and cracks. We studied the influence of different substrate materials like buffered $\mathrm{SiO}_{2}$, silicon and $\mathrm{Al}_{2} \mathrm{O}_{3}$, and various unbuffered crystall cuts of gadolinium gallium garnet (GGG) on the surface formation of BIG. The material had to be structured after the deposition. We examined structur- ing using plasma etching, laser structuring and deposition through masks. Then a waveguide was build and tested regarding its optical properties.

To optimize the light outcoupling from different microcavity OLED stacks we used numerical simulation. In the simulation we varied material and thickness of the metal anode, as well as the thickness of the hole and the electron conducting layers. The coupling of light into the garnets was also simulated. Afterwards selected microcavity OLED stacks were assembled and the electrical and optical properties were verified. In the end we combined microcavity OLED and garnet to form a magneto optical modulator.

2 Sample preparation Due to the reason that BIG is not thermodynamically stable [9], the use of liquid phase epitaxy normally used for growing garnets is not possible [10]. It is necessary to use non equilibrium processes such as Reactive Ion Beam Sputtering or Pulsed Laser Deposition (PLD) for its synthesis [11]. As already shown by Kahl et al. [12,13] Pulsed Laser Deposition (PLD) is a established method to create thin films of BIG.

All films for this paper were made using a standard laser ablation setup [5]. For detailed information about PLD please refer to [14-18].

We utilize a KrF excimer laser (Lambda Physik LPX $300)$ to generate UVlaser pulses ( $\lambda=248 \mathrm{~nm}, 30 \mathrm{~ns}$ ). These pulses then pass a lens and a rectangular shaped aperture to compensate the beam divergence and to blend out the inhomogeneous rim of the beam.

A second lens in front of the deposition chamber scales down the aperture and focuses the beam on the target. The target itself is inside a vacuum deposition chamber which the beam enters through a view port. The energy density on the target reaches up to $5 \mathrm{~J} / \mathrm{cm}^{2}$ (at $1.0 \mathrm{~J} /$ pulse). The high laser energy density on the target results in an evaporation of the target material and to a condensation of the material on the substrate which was located about $5 \mathrm{~cm}$ away from the target and placed on a resistive heater.

The atmosphere in the deposition chamber can be controlled by admitting a defined flow of process gas while constantly pumping the chamber. For being able to coat larger substrates as later needed for the OLED deposition the heater can be moved along the x-axis during the ablation. For an optimized process control the oxygen pressure $p_{\mathrm{O} 2}$, the heater current and voltage and the temperature of the sample holder $T_{H}$ were logged during the ablation process. Previous to the ablation process the substrates were cleaned in an ultrasonic bath of first acetone and second isopropyl alcohol, rinsed with pure water and then dried under nitrogen gas flow.

The PLD system was used to deposit yttrium iron garnet (YIG), bismuth iron garnet (BIG), indium tin oxide (ITO) and aluminium doped zinc oxide ( $\mathrm{ZnO}: \mathrm{Al})$. The optimized deposition parameters used are given in Table 1. 
Table 1 Deposition parameters used for BIG, YIG, ITO and $\mathrm{ZnO}$ :Al deposition. The laser pulse energy $E_{L}$ and frequency $\mathrm{f}_{L}$ had no effect on the film quality. During all processes their values were set to $E_{L}=650 \mathrm{~mJ}$ and $\mathrm{f}_{L}=8 \mathrm{~Hz}$

\begin{tabular}{ccc}
\hline Material & Temperature $\mathrm{T}_{H}\left({ }^{\circ} \mathrm{C}\right)$ & Pressure $\mathrm{p}_{O 2}$ \\
\hline BIG & 550 & $3.0 \times 10^{-2}$ \\
YIG & 700 & $3.0 \times 10^{-2}$ \\
ITO & 450 & $1.0 \times 10^{-2}$ \\
ZnO:Al & 500 & $3.0 \times 10^{-2}$ \\
\hline
\end{tabular}

All targets were prepared using sol-gel process. A detailed description of the sol-gel process is given in [6].

The OLEDs deposited for this work were all bottomemitting OLEDs. The OLEDs were either deposited on $140 \mathrm{~nm}$ thick industry standard ITO layers on glass, or on ITO deposited by PLD on various garnet stacks on fused silica. The ITO was either structured by chemical etching or by deposition through masks. An metal-only anode without ITO was used in some cases. A $30 \mathrm{~nm}$ layer of poly $(3,4)$-ethylendioxythiophene doped with poly(styrene sulfonate) (PEDOT:PSS) was spin cast on top of the ITO after an oxygen plasma treatment and dried on a hot plate.

The following deposition process was done in two major steps in a high vacuum chamber (base pressure $p<$ $3 \times 10^{-7}$ mbar) with a vacuum break in between. As all materials are deposited through shadow masks this is inevitable to rearrange the masks. The deposition chamber itself is connected to a glove box with nitrogen atmosphere to prevent contamination and degradation of the samples during the vacuum break and afterwards.

The bottom electrode consisting of $15 \mathrm{~nm}$ to $30 \mathrm{~nm}$ silver is evaporated in the first deposition step. Then a $1 \mathrm{~nm}$ layer of tetrafluorotetracyanoquinodimethane (F4TCNQ) was applied on top to optimize the hole injection. As hole transporter $N, N^{\prime}$-diphenyl- $N, N^{\prime}$-bis(3methylphenyl)-1,1'-biphenyl-4,4-diamine (TPD) with a thickness of $80 \mathrm{~nm}$ to $300 \mathrm{~nm}$ was used. The emitter was tris-(8-hydroxyquinoline) aluminium $\left(\mathrm{Alq}_{3}\right)$ with a layer thickness of $80 \mathrm{~nm}$. $\left(\mathrm{Alq}_{3}\right)$ has a photoluminescence peak at $535 \mathrm{~nm}$ and therefore fits very well to the $532 \mathrm{~nm}$ were the Faraday-rotation of BIG has its maximum. All organic materials were deposited using effusion cells.

The chamber was vented with nitrogen next, prepared for the second phase and pumped down till it reached the base pressure. Then the cathode composed of a calcium and a aluminium layer was deposited. The OLED was encapsuled after the deposition using compound glue and a glass slide. Further details to the OLEDs characterization are given in Section 7.

3 Garnet morphology OLEDs are ultrathin devices with a thickness of $200 \mathrm{~nm}$ to $500 \mathrm{~nm}$. Thus the roughness of the substrates for OLED deposition should be as low as possible. As we want to deposit the OLED on garnet material a smooth, crack free garnet surface is desirable.

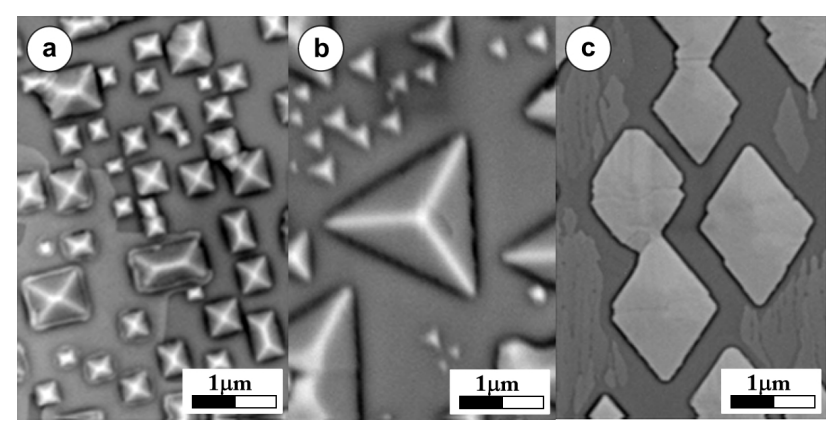

Figure 2 BIG growth on various GGG cuts. a: BIG on GGG 100, b: BIG on GGG 111, C: BIG on GGG 110.

It is only possible to grow BIG on a garnet understructure. Figure 2 shows the initial growth on different GGG cuts as reported by [8]. BIG shows island growth on all substrates, adapting to the underlying structure. BIG grown on GGG (110) (Fig. 2c) has the less rough surface. Therefore we want to compare the roughness for thick BIG films grown on various GGG substrates in section 3.1. An overview over the properties of the used substrates is given in Table 2.

Table 2 Lattice constant and orientation of different substrates.

\begin{tabular}{cccc}
\hline Label & Material & Orientation & Lattice const. \\
\hline GGG & $\mathrm{Gd}_{3} \mathrm{Ga}_{5} \mathrm{O}_{12}$ & $(100)$ & $1.238 \mathrm{~nm}$ \\
& & $(110)$ & $1.238 \mathrm{~nm}$ \\
& & $(111)$ & $1.238 \mathrm{~nm}$ \\
$\mathrm{~S} * \mathrm{GGG}$ & $\mathrm{Ca}, \mathrm{Mg}, \mathrm{Zr}$ & $(110)$ & $1.243 \mathrm{~nm}$ \\
& doped GGG & & \\
SGGG & $\mathrm{Gd}_{2.65} \mathrm{Ca}_{0.35} \mathrm{Ga}_{4.05}$ & $(110)$ & $1.250 \mathrm{~nm}$ \\
& $\mathrm{Mg}_{0.30} \mathrm{Zr}_{0.65} \mathrm{O}_{12}$ & & \\
\hline
\end{tabular}

Beside the possibility of growing BIG on GGG bulk material it is possible to employ a buffer system on non garnet material [6]. We analyzed GGG and YIG buffer systems to optimize the roughness of the garnet stack. In both systems cracks appear which have to be removed. Therefore we utilized different substrates with different thermal expansion coefficients to reduce the stress at the substrate garnet interface and thus reduce cracks.

3.1 Substrate influence Figure 3 shows ESEM images of BIG grown on GGG (100), GGG (110), $S *$ GGG (110) and SGGG(110). The pictures were taken using BSE detector while a $\mathrm{H}_{2} \mathrm{O}$ background pressure of 0.3 Torr was applied. All films were grown together in one deposition step using standard parameters for BIG as given in Table 1. The films showed no cracks in all magnifications.

Figures $3 \mathrm{~b}$ ) and c) exhibit nearly the same morphology with wormlike textures of about $250 \mathrm{~nm}$ length, while 3 a) grown on GGG (100) has a more randomly oriented surface. In Fig. 3 d) the structures shown in b) and c) become more oriented. It also show the texture which can be 


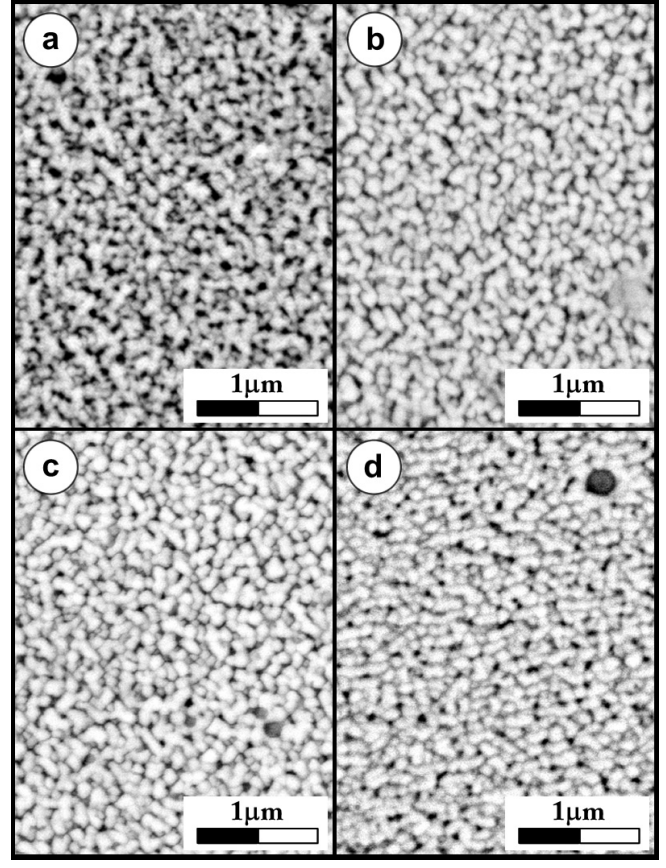

Figure $3248 \mathrm{~nm}$ BIG on a) GGG (100), b) GGG (110), c) S*GGG (110) and d) SGGG(110).

observed in Fig. 3 b) and c) but roughly aligned in bands. None of the structures shown in Fig. 2 can be found on the film surface.

A Cross-section TEM image of a BIG film on GGG explains these observations (Fig. 4). After the nucleation of BIG on the GGG surface in the appropriate structure the islands start to grow lateral. During the first $50 \mathrm{~nm}$ the growth structure is largely disturbed due to mutual overgrowth. Larger grains are formed in that phase. From

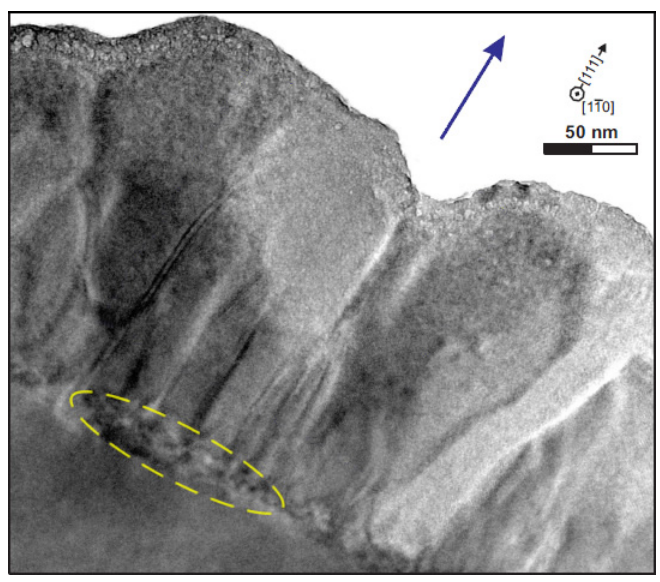

Figure 4 TEM cross-section image of $200 \mathrm{~nm}$ BIG on GGG (111). The yellow ellipse marks a part of the interface between GGG and BIG. The adaption of BIG to GGG leads to a high disorder near the interface. that point on the growth mode is more determined by the plasma and the temperature then the substrates structure, which leads to a similar morphology in all processes.

The aspired change in the growth mode of BIG therefore seems to have no significant influence on the surface roughness of BIG. AFM data was obtained from the samples to verify this. The results are given in Table 3

Table 3 AFM measurements of $248 \mathrm{~nm}$ BIG deposited on GGG (100), GGG (110), S*GGG (110) and SGGG(110).

\begin{tabular}{ccc}
\hline Material & $\mathrm{R}_{a}(\mathrm{~nm})$ & $\mathrm{RMS}(\mathrm{nm})$ \\
\hline GGG $(100)$ & $\mathrm{xxxx}$ & \\
GGG $(110)$ & & \\
S*GGG (110) & & \\
SGGG (110) & &
\end{tabular}

3.2 Buffered growth As shown by Koerner et al. [6] it is possible to deposit BIG on non garnet substrates by using buffer layers. Problems that arise are cracks in the film due to the different thermal expansion coefficients of garnet and substrate. We analyzed two different buffers based on GGG and YIG on fused silica, silicon and aluminium oxide to reduce cracks and roughness of the buffer and of the garnet surface. An overview over the different coefficients of thermal expansion for these materials is given in Table 4.

\begin{tabular}{ccc}
\hline Material & $\alpha_{\text {thermal }} \frac{1}{\circ} \mathrm{K}$ & lattice constant \\
\hline \hline Silicon & $2.6 \times 10^{-6}$ & $5.43 \AA$ \\
$\mathrm{SiO}_{2}$ & $0.5 \times 10^{-6}$ & amorph \\
$\mathrm{Al}_{2} \mathrm{O}_{3}$ & $8.0 \times 10^{-6}$ & $\mathrm{a}=4.76 \AA$ \\
& & $\mathrm{c}=13.00 \AA$ \\
$\mathrm{Gd}_{3} \mathrm{Ga}_{5} \mathrm{O}_{12}$ & $9.2 \times 10^{-6}$ & $12.38 \AA$ \\
$\mathrm{Y}_{3} \mathrm{Fe}_{5} \mathrm{O}_{12}$ & $10.4 \times 10^{-6}$ & $12.37 \AA$ \\
$\mathrm{Bi}_{3} \mathrm{Fe}_{5} \mathrm{O}_{12}$ & $12.9 \times 10^{-6}$ & $12.63 \AA$ \\
\hline
\end{tabular}

Table 4 Coefficients of thermal expansion for silicon, fused silica, aluminium oxide, GGG, YIG and BIG. Garnet coefficients are taken from Chern et. al.[19].

GGG and YIG were deposited on silicon, fused silica and aluminium oxide using the parameters given in Table 1 . The films were tempered at $1000^{\circ} \mathrm{C}$ for $3 \mathrm{~h}$ under oxygen gas flow afterwards. A complete characterization of the surface parameters was done utilizing ESEM and AFM. Afterwards BIG was applied on top of the buffers. The films were then characterized again. In Fig. 5 the obtained ESEM images of buffer and BIG are shown. The corresponding roughness values are given in Table 5.

Both GGG and YIG buffers show cracks on fused silica and silicon whereas the buffer tiles are smaller on $\mathrm{SiO}_{2}$ then on $\mathrm{Si}$. The reason therefore is tensile stress in the film. The film is tempered at $1000^{\circ} \mathrm{C}$ and cools down to room temperature afterwards. Thus the film shrinks. As the thermal expansion coefficients for YIG and GGG are higher 

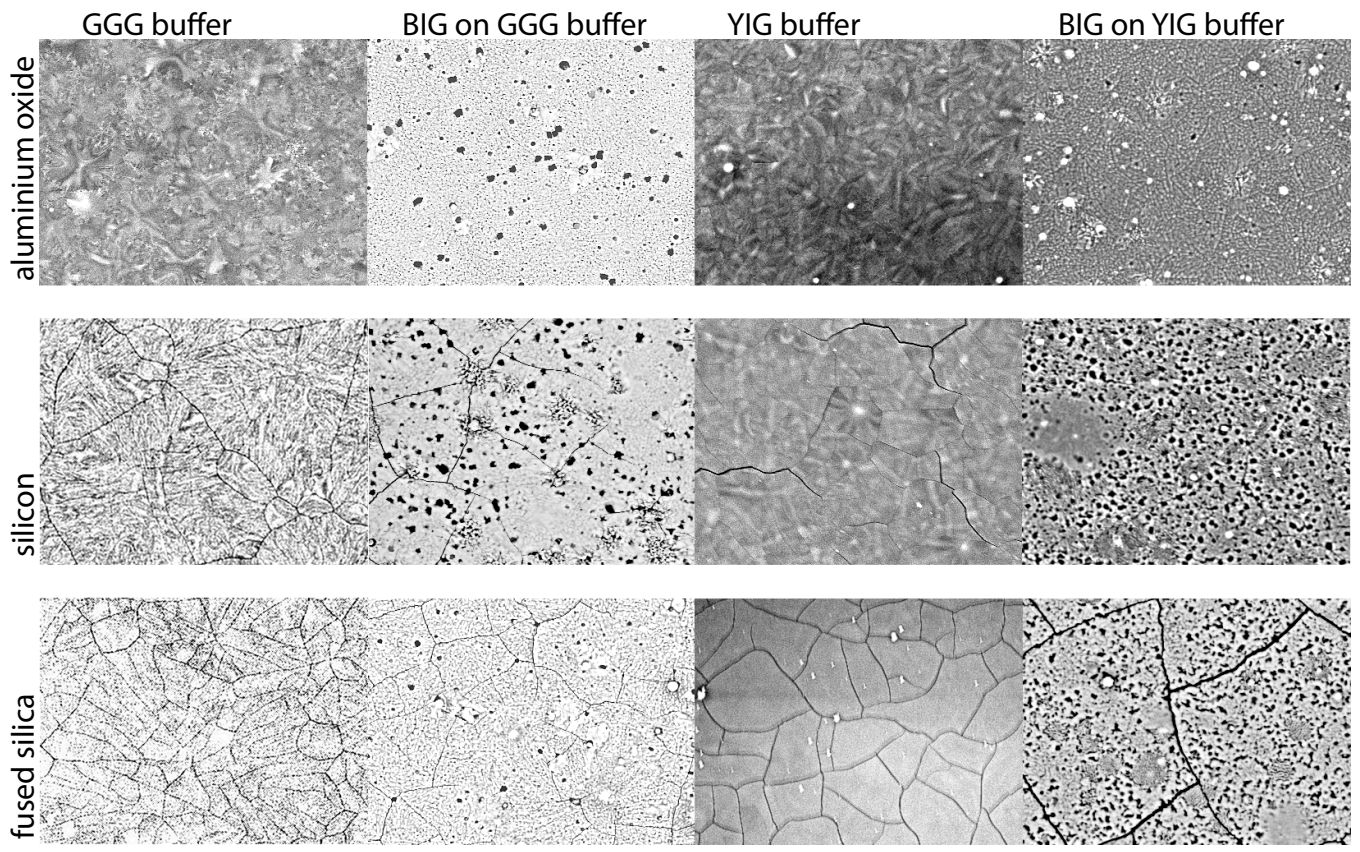

Figure 5 YIG and GGG buffers with and without BIG. One image equates to a width of $4 \mu \mathrm{m}$ and an height of $3 \mu \mathrm{m}$. First row: aluminium oxide, second row: silicon, third row: fused silica. First column: GGG buffer, second column BIG on GGG buffer, third column: YIG buffer, forth column: BIG on YIG buffer. Buffer thickness $d_{\text {Buffer }}=25 \mathrm{~nm}$, BIG thickness $d_{\text {BIG }}=820 \mathrm{~nm}$.

than those of the substrate this leads to tensile stress. The thermal expansion coefficient of $\mathrm{SiO}_{2}$ is lower than the one of silicon and thus leads to higher strain and for this reason to smaller buffer tiles. The buffers deposited on $\mathrm{Al}_{2} \mathrm{O}_{3}$ show no cracks at all.

The BIG grown on the buffers afterwards overgrows some of the cracks and forms larger tiles. This originates in substrate temperature of only $550^{\circ} \mathrm{C}$ during the BIGs deposition. The, compared to the tempering step, much lower

Table 5 AFM measurements of BIG deposited on YIG and GGG buffers on aluminium oxide $\left(\mathrm{Al}_{2} \mathrm{O}_{3}\right)$, silicon $(\mathrm{Si})$ and fused silica $\left(\mathrm{SiO}_{2}\right)$ substrates.

\begin{tabular}{lcrrr}
\hline Substrate & Buffer & \multicolumn{1}{l}{ BIG } & $\mathrm{R}_{a}(\mathrm{~nm})$ & RMS (nm) \\
\hline $\mathrm{Al}_{2} \mathrm{O}_{3}$ & GGG & & 0.87 & 1.04 \\
$\mathrm{Si}$ & GGG & & 1.52 & 2.05 \\
$\mathrm{SiO}_{2}$ & GGG & & 2.72 & 3.50 \\
$\mathrm{Al}_{2} \mathrm{O}_{3}$ & YIG & & 0.70 & 0.95 \\
$\mathrm{Si}$ & YIG & & 1.42 & 1.78 \\
$\mathrm{SiO}_{2}$ & YIG & & 1.67 & 2.28 \\
$\mathrm{Al}_{2} \mathrm{O}_{3}$ & GGG & $820 \mathrm{~nm}$ & 11.69 & 15.64 \\
$\mathrm{Si}_{\mathrm{SiO}}$ & GGG & $820 \mathrm{~nm}$ & 24.17 & 30.09 \\
$\mathrm{Al}_{2} \mathrm{O}_{3}$ & GGG & $820 \mathrm{~nm}$ & 14.46 & 18.66 \\
$\mathrm{Si}_{\mathrm{SiO}}$ & YIG & $820 \mathrm{~nm}$ & 8.29 & 10.61 \\
$\mathrm{SiO}_{2}$ & YIG & $820 \mathrm{~nm}$ & 23.14 & 29.18 \\
\hline
\end{tabular}

temperature difference leads to less stress in the film which thus resolves over larger areas.

The roughness values obtained from AFM measurements are given in Table 5. The YIG buffers are less rough compared to the GGG buffers. Also the BIG films deposited on YIG exhibit a lower roughness. This is due to grain formation in the buffer layers. In the GGG buffer the grains grow slightly faster. This leads to a higher roughness of the GGG buffer.

All BIG films grown on YIG or GGG buffered silicon show a considerable higher roughness. This can be due to higher formation energy of nuclei on the surface, which leads to larger grains on thus to a higher roughness as suggested by Park et. el. [20]. This originates in the occurrence of interface effects during the tempering step under oyxgen atmosphere. During the 3 hour tempering step at $1000^{\circ} \mathrm{C}$

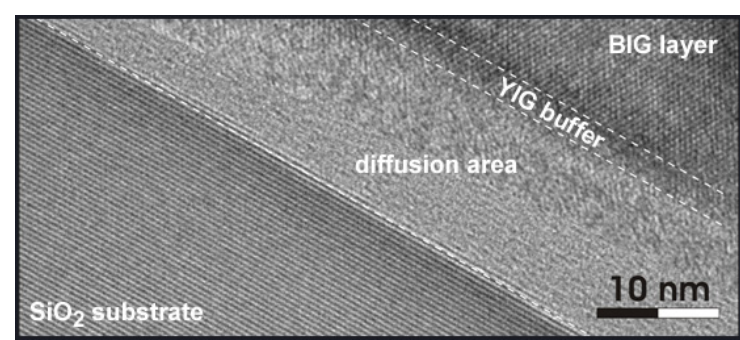

Figure 6 Cross-section TEM image of BIG on a YIG buffered $\mathrm{SiO}_{2}$. 
the topmost $100 \mathrm{~nm}$ of the Silicon substrate are oxidised to $\mathrm{SiO}_{2}$ [21,22]. This leads to a change in buffer nucleation and thus to a higher roughness. The $\mathrm{SiO}_{2}$ and the $\mathrm{Al}_{2} \mathrm{O}_{3}$ are thermal stable at $1000^{\circ} \mathrm{C}$. An approximately $15 \mathrm{~nm}$ thick layer between buffer and substrate is affected by diffusion processes from the buffer into the substrate. A TEM image showing the buffer-substrate interface is shown in Fig. 6 . The area where the YIG buffer diffuses into the fused silica substrate is clearly visible.

4 Structuring of garnets To build a garnet based waveguide the material has to be structured after the deposition. We focused on Laser structuring and Ion Beam Etching as structuring techniques.

It has been demonstrated in the past years that femtosecond lasers are excellent tools for the micro structuring of nearly all kinds of solid materials [23-27]. We utilized this technique for the structuring of GGG and YIG buffered BIG films. The laser structuring was done in cooperation with the Research Centre for Microtechnology at the Vorarlberg University of Applied Sciences [28]. A diode pumped solid-state laser (HighQLaser, FemtoREGEN) emitting pulses at a wavelength of $1040 \mathrm{~nm}$ with a pulse duration of $350 \mathrm{fs}$ and a repetition rate of up to $100 \mathrm{kHz}$ was employed. The beam track on the sample was controlled by a laser scanner. The intensity of the beam was adjusted with a combination of a half-wave plate and a polarizer.

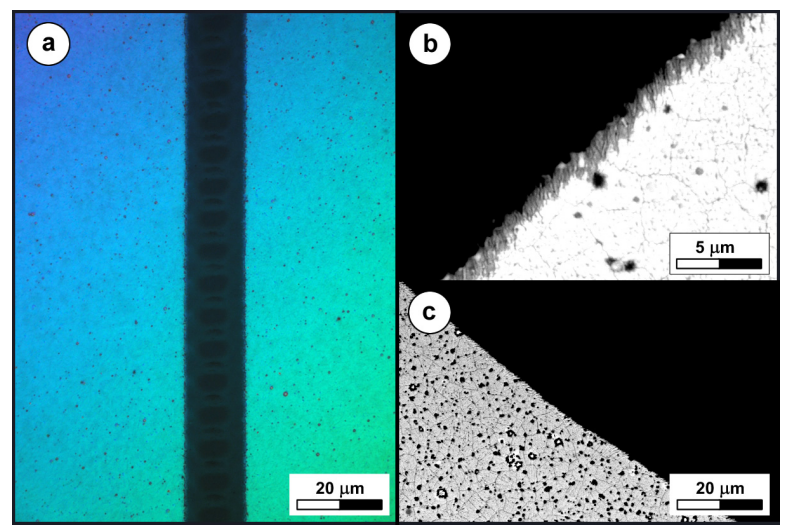

Figure 7 Laser structured $\mathrm{BIG}$ on $\mathrm{SiO}_{2}$. a) differential interference contrast microscopy image of a trench cut by the femtosecond laser, b) and c) ESEM images of the border of a circular structure with a radius of $500 \mu \mathrm{m}$.

A series of experiments was done to optimize the structuring process. The best results were obtained for a power of 0.8 Watt, a laser repetition rate of $100 \mathrm{kHz}$ and a scan speed of $1 \mathrm{~m} / \mathrm{s}$. This process was repeated 16 times to get a better distribution of the pulses along the scan path. ESEM images of structured BIG are shown in Fig. 7. Figure 7a shows differential interference contrast microscopy images of a trench cut into YIG buffered BIG on $\mathrm{SiO}_{2}$. The trench is $15 \mu \mathrm{m}$ wide and cuts through the BIG film into the substrate. Figure $7 b$ and $c$ show ESEM images of the border of a circular cut structure with a radius of $500 \mu \mathrm{m}$. The black area is the $\mathrm{SiO}_{2}$ substrate. The edge of the garnet is $2 \mu \mathrm{m}$ wide and sloped from the top edge to the bottom. It exhibits a lateral roughness of $250 \mathrm{~nm}$.

We also examined the capability of Ion Beam Etching for garnet structuring. Photoresist covered the parts of the sample which should not be removed. Argon ions with energy of $500 \mathrm{eV}$ were used to etch the uncovered parts of the surface. Higher ion energys lead to a degeneration of the photoresist. As the etching rate for the photoresist is 4 times higher than for the garnet the photoresist has to be at least $4 \mu \mathrm{m}$ thick. This leads to problems with the spin coating process and the developing. The result of an Ion Beam Etching process on YIG-buffered BIG is shown in Fig. 8.

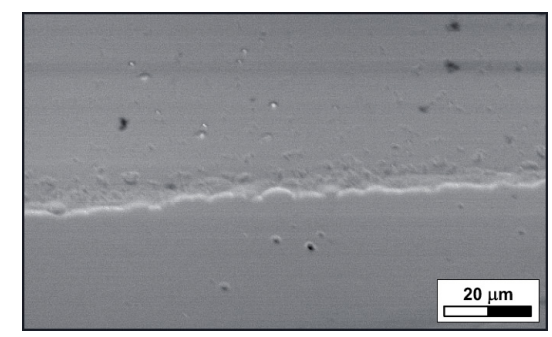

Figure 8 Ion beam etched YIG buffered $\mathrm{BIG}$ on $\mathrm{SiO}_{2}$.

The overall quality of the structuring is worse compared to Laser structuring. The edge of the etched sample exhibits a high waviness, the process takes unequally longer and the preparation is more complex. The results of the Laser structuring are good. It is done without complex preparations within minutes and thus preferable. The results are comparable to the results obtained for e.g. buried channel waveguides [29]. Lee et al. [30] associates the transmission loss of a waveguide with the waveguide width. They conclude that a wide waveguide strongly reduces the loss due to random wall imperfections. As the lateral dimensions of our waveguide are four magnitudes higher ( 3 to $6 \mathrm{~mm}$ ) than the wall roughness of $250 \mathrm{~nm}$ the loss at the waveguide walls is neglectable.

5 Optical properties of BIG Garnets are well known for their high optical quality and Faraday-rotation in the infrared, but modern applications for BIG are focused on the visible wavelength. Especially the use of BIG as material for magnetooptical sensors set the focus to the Faraday-rotation peak around $532 \mathrm{~nm}$ wavelength [31,32]. In Fig. 9 the Faraday-rotation for BIG films of various thicknesses is shown. For thin films a positive rotation maximum around $420 \mathrm{~nm}(2.95 \mathrm{eV})$ can be observed. Here the highest Faraday-rotation per $\mu m$ of up to $60 \% / \mu m$ can be obtained. For films thicker than $200 \mathrm{~nm}$ this peak cannot be measured anymore due to the high absorption in BIG. The second remarkable point is the maximum of the 


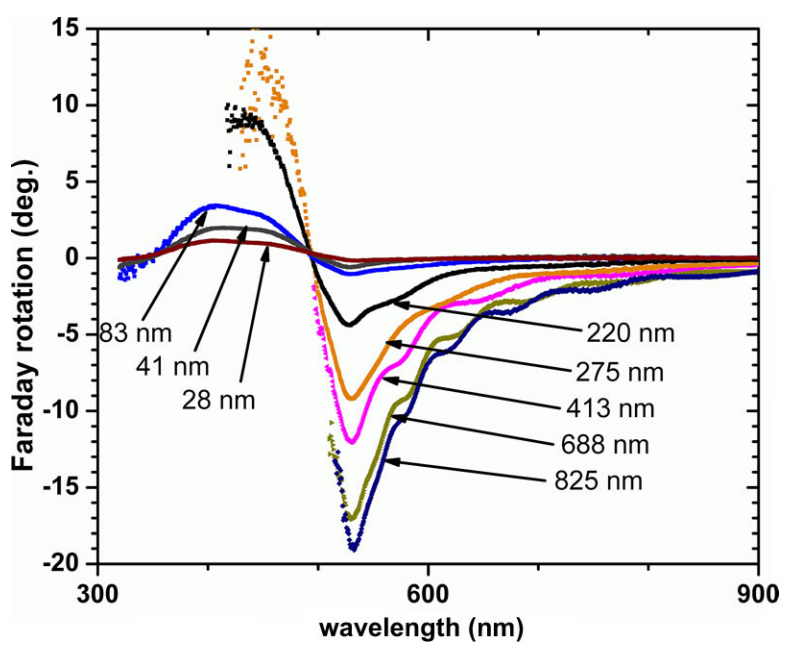

Figure 9 Faraday-rotation of BIG for various film thicknesses. The data is shown for a transmission greater than $0.1 \%$. The Transmission is given in Fig. 10.

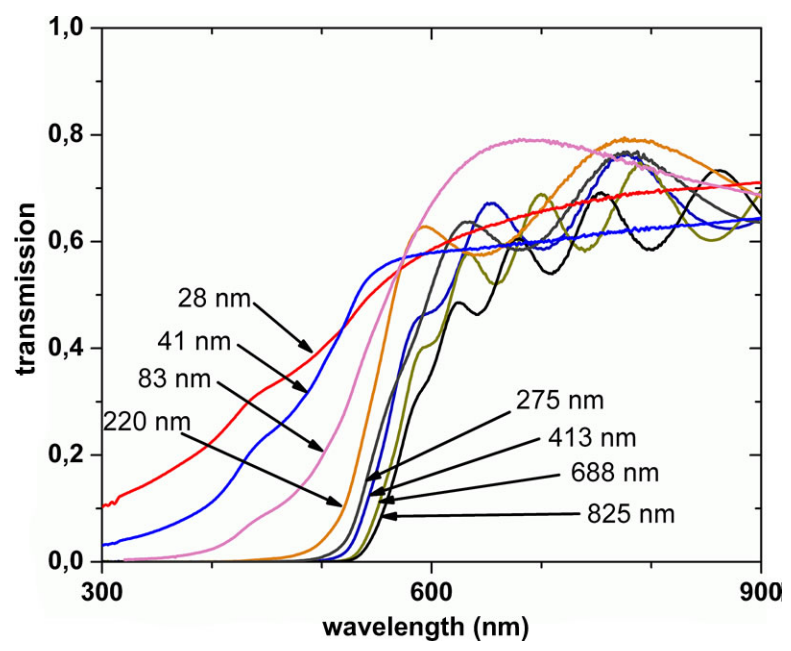

Figure 10 Transmission of BIG films for various film thicknesses. A transmission of 1 corresponds to a total transmission of $100 \%$.

negative Faraday-rotation around $530 \mathrm{~nm}(2.34 \mathrm{eV})$. Here the Farady-rotation reaches values of more than $25^{\circ} / \mu \mathrm{m}$ in BIG.

This is a result of structure of the iron garnets. $\mathrm{Fe}^{3+}$ occupies the tetrahedral and the octahedral sites of the garnet structure forming two sub lattices with an opposite magnetization causing ferrimagnetic behavior. Between these $\mathrm{Fe}^{3+}$ ions crystal field transitions are possible which interact with right circulary polarized light (RCP) and left circulary polarized light (LCP) in different ways [33]. Also transitions between the orbital singlet ground state and an exited state split by the effect of exchange field and and spin-orbit coupling are possible. Wittekeok et al. [34] identified two strong optical transitions at $2.8 \mathrm{eV}$ and $3.3 \mathrm{eV}$ as

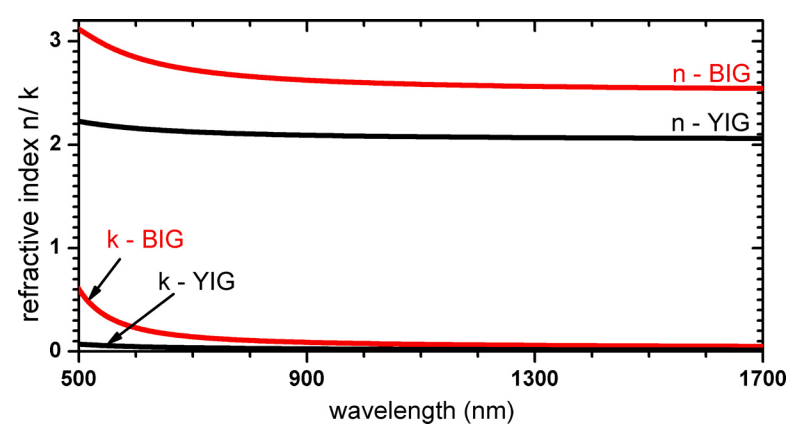

Figure 11 Optical constants of YIG and BIG determined by ellipsometric measurements.

beeing mainly responsible for the remarkable increase of the Faraday-rotation in the visible and the near infrared. A third transition around $2.5 \mathrm{eV}$ is responsible for the observed minimum. The incooperation of Bismuth has an strong effect on the exchange field and the spin-orbit coupling and thus leads to the high Faraday-rotation in BIG [35-38].

To determine the optical constants of YIG and BIG ellipsometric measurements were done using a standard ellipsometer (Sentech SE850). The measured data was fit using an oscillator layer with no free charge carriers for the garnets. We compared our results to data obtained from transmission measurements [6] and found them in good agreement. An overview over the obtained optical constants of YIG and BIG is given in Fig. 11.

6 Microcavity OLED simulation In standard organic light-emitting device (OLED) structures a large fraction of the generated light is trapped inside the organic layers or the substrate by total internal reflection. This fraction is lost by reabsorption or emission at the device edges. A rough estimate shows that only about $20 \%$ of the generated photons can leave the device in forward direction [39-43]. As is normally desired to raise this fraction of light by e.g. employing micro lenses or scattering microspheres at the backside of the glass substrate [44,45], this is not aspired for our case. The essential idea in this context is to incorporate a garnet layer in the substrate to form a magnetooptical waveguide and thus utilize the trapped light. Moreover, in OLEDs with a semitransparent metall anode, this anode is forming a microcavity resonator together with the counter electrode, which usually is a fully reflective cathode. The light emission from microcavity OLEDs is spectrally narrowed and displays a strong angular dependence of the peak wavelength [46-48]. The emission angle can be adjusted by changing the layer thickness of the employed organic films. It has also been observed that the emission peak of microcavity OLEDs can be shifted up to $100 \mathrm{~nm}$ in the optical range [49-52]. By shifting the emission angle for light of $532 \mathrm{~nm}$ wavelength to values above $41.5^{\circ}$ the light cannot leave the substrate anymore. 
To optimize the emission characteristics of microcavity OLEDs we used optical simulations. The optical modeling of OLEDs must consider near-field phenomena and the photonic mode density due to the use of thin organic films. It is most common to use the dipole model developed by Chance, Prock and Silbey [53,54] for molecular fluorescence and energy transfer near interfaces as base for simulations. This model was extended to the nearfield optics of OLEDs by various authors [55-59], where an exciton within the OLED is modeled as a point dipole driven by the reflected electromagnetic waves inside a microcavity. The simulations presented in this work are also based on this traditional approach of emissive dipoles. The dipoles, embedded in the multilayer stack of an OLED, are treated as forced damped harmonic oscillators $[55,56]$. The orientation of the emitting molecules is taken to be isotropic. Furthermore, the radiative quantum efficiency of Alq 3 is taken to be $20 \%$. For more information about the actual implementation please refer to Nowy et. al. [60].

In the first simulation we comprehended the transition from a basic OLED structure as shown in Fig. 1a to the microcavity device in Fig. 1b. As additional anode materials on top of ITO layers of silver Ag, platinum Pt, nickel Ni and gold Au have been simulated. The fraction of power coupled into the substrate is given in Fig. 12. Silver exhibits the best results. The light coupled into the substrate even rises with increasing Ag thickness. All other materials show a decline in the out coupled power caused by absorption in the material or deficient reflectivity.

In Fig. 13 the emission characteristics for a microcavity OLED containing a silver mirror are shown. The total emission is the sum of emission to the substrate and the direct emission. While the direct emission declines with a growing silver film thickness, the emission to the substrate rises disproportionately high. Figure 14 shows the simulated power dissipation spectra appertaining to the chosen silver film thicknesses. The spectra show the power cou-

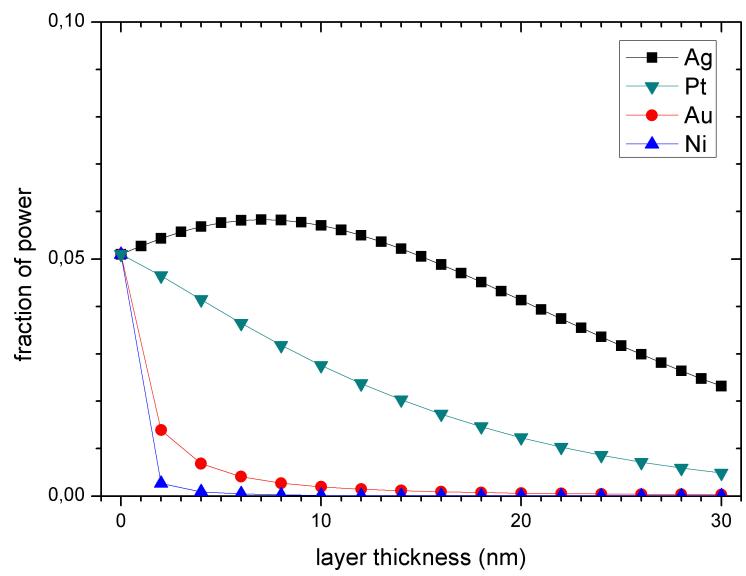

Figure 12 Simulation of OLEDs emission characteristics for various metals embedded between ITO and TPD as shown in Fig. 1b.

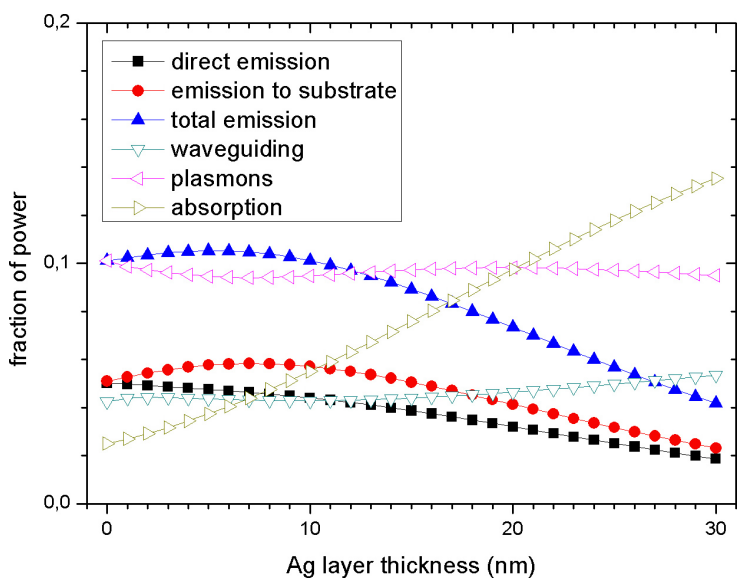

Figure 13 Simulation of OLEDs emission characteristics for an $\mathrm{Ag}$ layer embedded between ITO and TPD as shown in Fig. 1b. The total emission is the sum of emission to the substrate and the direct emission. Waveguiding refers to the amount of light guided in the organic layers.

pled from the OLED into the substrate. Light coupled in with an angle lower than $41.5^{\circ}$ is out coupled (direct emission). The shown spectra are spectrally narrowing with thicker Ag layers and the maximum emission rises. Its peak emission is located between $12 \mathrm{~nm}$ and $18 \mathrm{~nm} \mathrm{Ag}$ thickness around $48^{\circ}$ and $549 \mathrm{~nm}$. The emission to the substrate shown in Fig. 13 has its maximum around $7 \mathrm{~nm}$. The reason for this is a sharpening of the emission peak with rising Ag thickness while the total emission declines. Also the p-polarized light emission is reduced and thus lessens the combined s- and p-polarized emission to the substrate.

The absorption in the OLED is rising with the film thickness while the number of plasmons and the waveguiding in the organic layers is nearly constant. There is no influence on the position of the emission maximum. The position of the emission maximum can be changed by altering the organic layers thickness. This changes the distance between the dipoles and the front (anode) and back (cathode) mirror. In Figure 15 the organic layer thickness has been varied. The thickness of TPD and $\mathrm{Alq}_{3}$ are always the same. The given value is total thickness of the organic layer with the dipole location in the exact middle. The image shows the change in the coupling of light into the substrate. A shift of the emission in angle and wavelength is observable.

Using the acquired simulation data it was possible to optimize the emission into the substrate. Best results were obtained for $\mathrm{a} \mathrm{Alq}_{3}$ and TPD layer thickness of $80 \mathrm{~nm}$. To compensate the roughness thick TPD layers give better results. The angular resolved spectral emission for a TPD layer thickness of $295 \mathrm{~nm}$ is also appropriate.

7 Microcavity OLED deposition To verify the obtained simulation data selected OLEDs were deposited. Afterwards the voltage-current characteristics and the angu- 


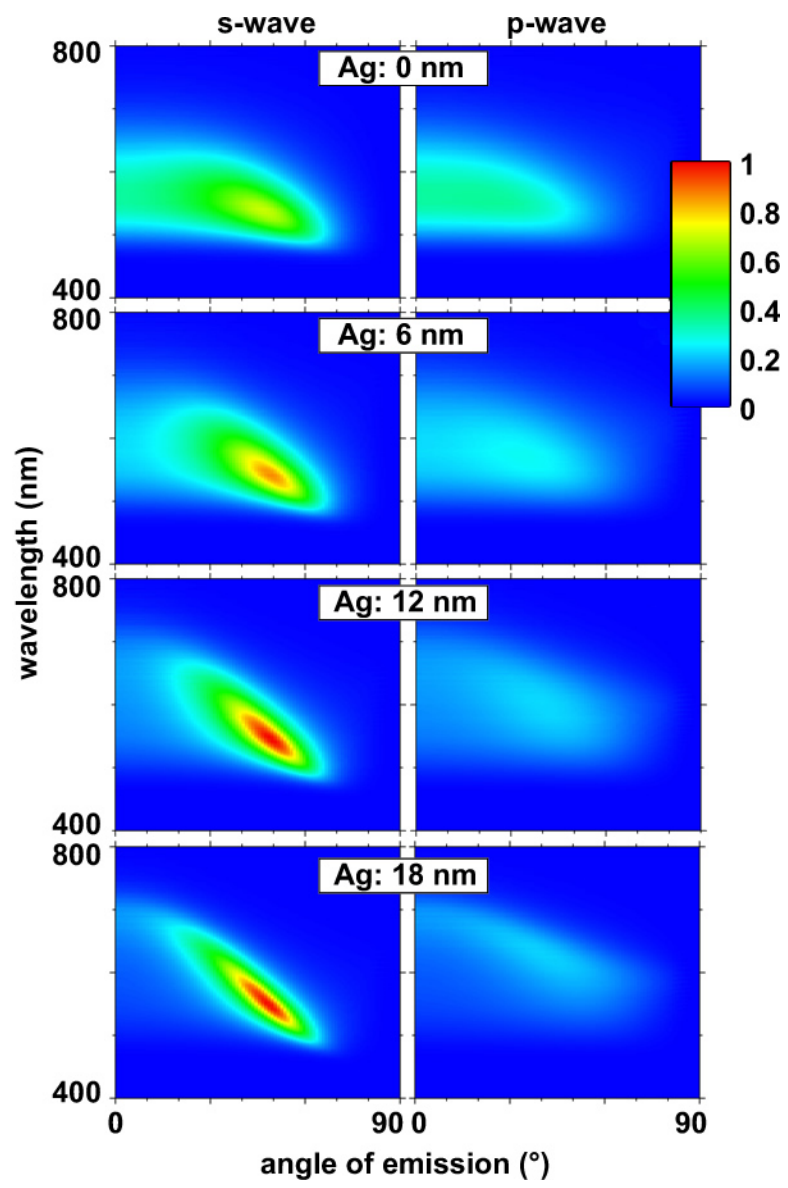

Figure 14 Simulated power dissipation spectra for selected $\mathrm{Ag}$ film thicknesses. The spectra show the power coupled into the substrate. Only light coupled into the substrate at an angle greater than $41.5^{\circ}$ stays in the substrate. All spectra have been normalized to the same factor.

lar dependent emission spectra were measured. To gain the emission into the substrate a semicircle prism was attached to the OLED using index-matching gel. It was mounted on a rotary table so that the axis of rotation was coincident with the active area of the devices and s- and p- polarized emission was measured consecutively. For the measurements of the electroluminescence spectra the samples were driven at a constant current using a Keithley 220 current source. The setup is shown in Fig. 16.

Figure 17 shows current and luminance of a typical microcavity OLED. The OLED shows a good diode characteristic with low leakage current. The measurement was done from +10 Volt to -10 Volt and reverse. The diode characteristic of the microcavity OLED can be observed very well. Luminescence begins with the charge carrier injection at $2.5 \mathrm{~V}$. The obtained luminance values are comparable to non cavity OLEDs.

The associated angular dependent emission spectra of the emission into the substrate together with the simulated

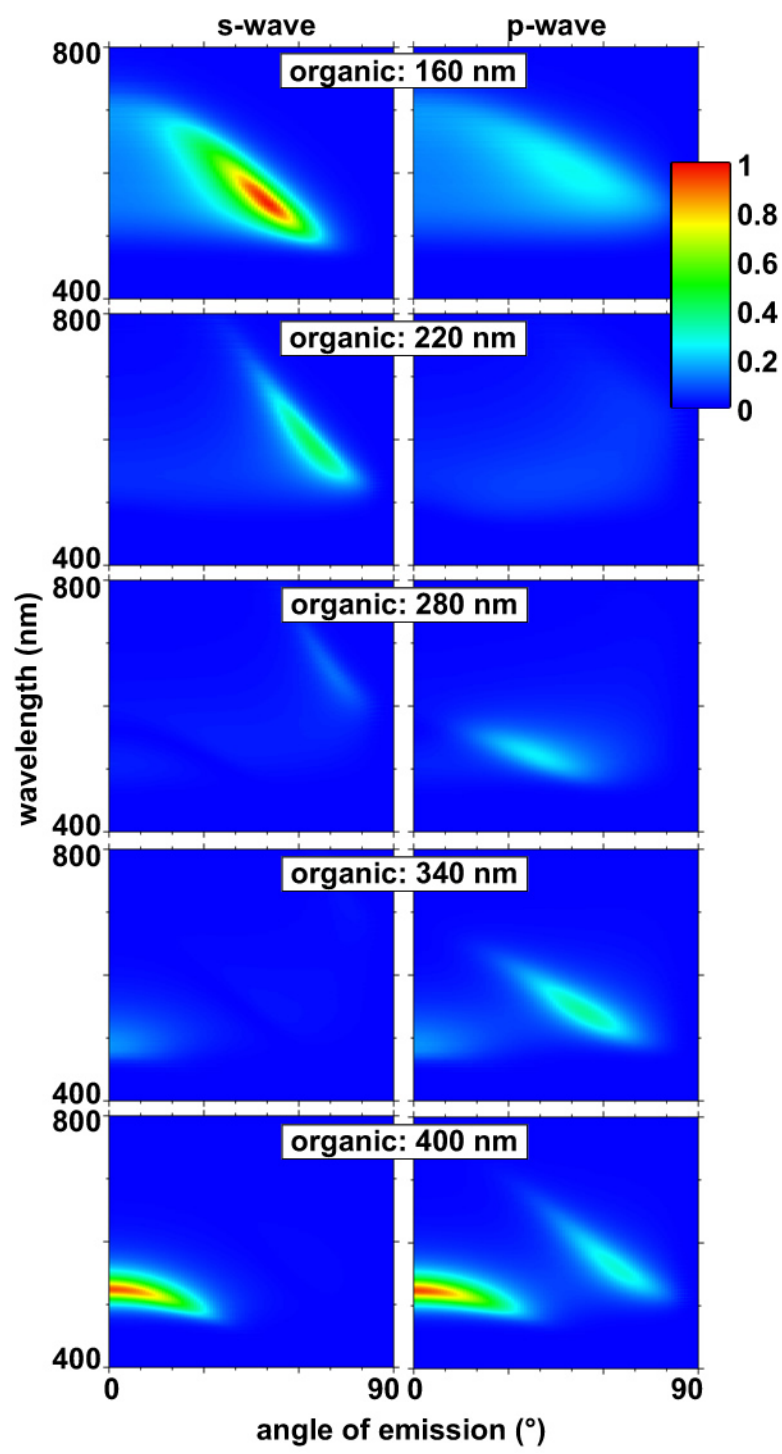

Figure 15 Coupling of light into the substrate. The given organic thickness is the total organic layer thickness for TPD plus $\mathrm{Alq}_{3}$. The thickness of an single TPD or $\mathrm{ALQ}_{3}$ layer is the given thickness divided by 2 . All spectra have been normalized to the same factor. The Ag layer thickness is $10 \mathrm{~nm}$.

values are given in Fig. 18. The figures are normalized with respect to the highest intensity obtained in each of them. Corresponding s- and p-polarized spectra are normalized with the same factor. The simulation and the measured values are in good agreement.

8 Optical modulation As our ambition was to build an integrated optical modulator a final setup including all components had to be assembled. Therefore we decided to build a planar waveguide containing a garnet core. As the absorption in the garnet material in the optical range is not optimal the thickness of the garnet has to be adjusted 


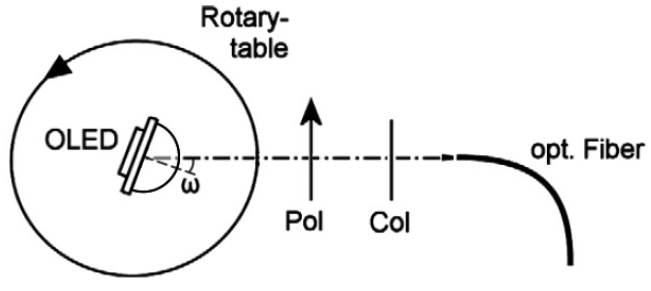

Figure 16 Setup for measuring the angular dependent emission spectra of OLEDs. The OLED is attached to a semicircle prism using index-matching gel. It is mounted on a rotary table and light emission is investigated with a collimator and a polarizing filter.

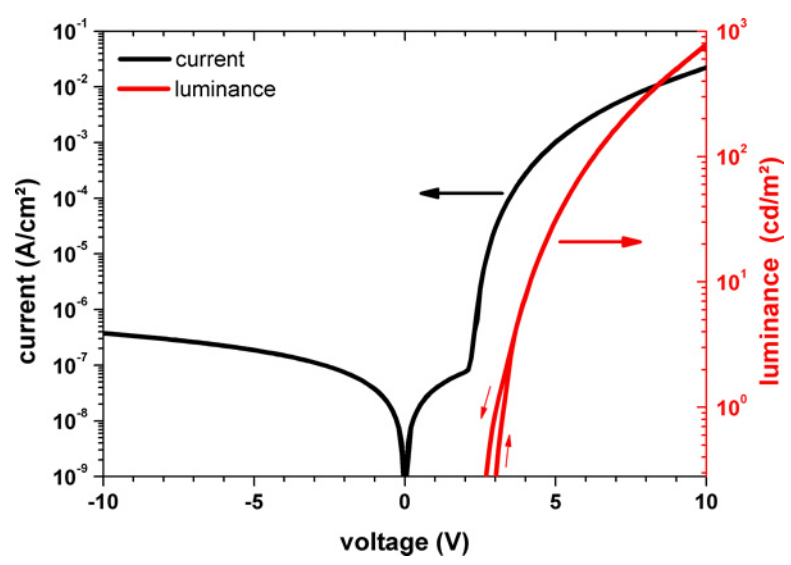

Figure 17 Current and luminance of a typical microcavity OLED. The measurement was started at +10 Volt.

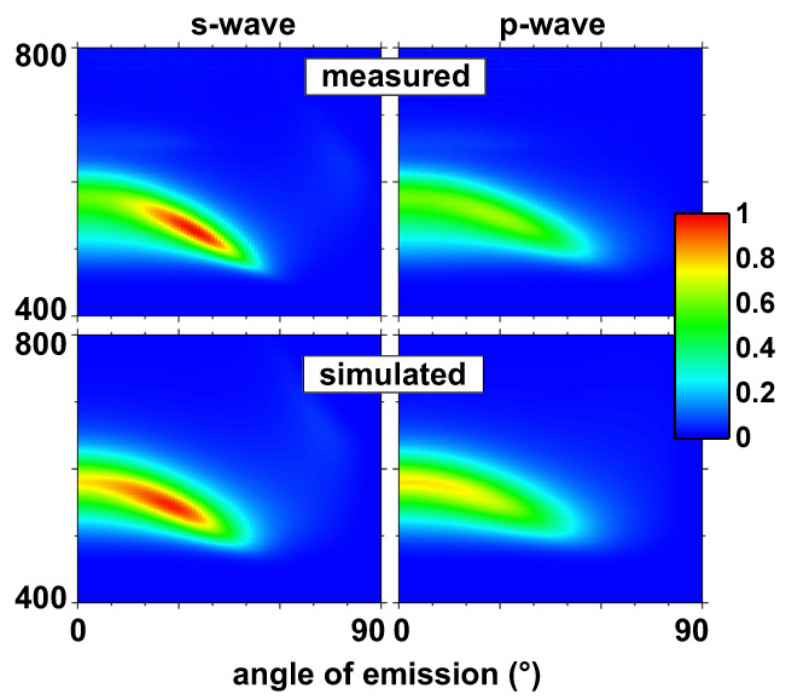

Figure 18 Simulated and measured angular dependent emission spectra of microcavity OLEDs.

to the desired waveguide length and Faraday-rotation. This is possible using an embedded garnet core. The OLED is placed directly on $\mathrm{SiO}_{2}$ of the waveguide. The light is mod- ulated by crossing the garnet layer. As BIG has a high refractive index of $\mathrm{n}>2.8$ at $532 \mathrm{~nm}$ the light is not trapped inside or reflected on the layer. A prism is used to outcouple the light into a spectrometer. The spectrometer is placed behind a polarizer. A principal layout of the setup is shown in Fig. 19. The demonstrator was build using a 125 nm high garnet core in a $1000 \mu \mathrm{m}$ high $\mathrm{SiO}_{2}$ waveguide

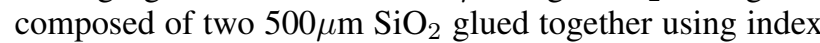
matching gel. One of the slides was coated with a YIGbuffered BIG before. The OLED was deposited directly on top of the waveguide. The light from the OLED was guided about a length of $5 \mathrm{~mm}$ to the prism.

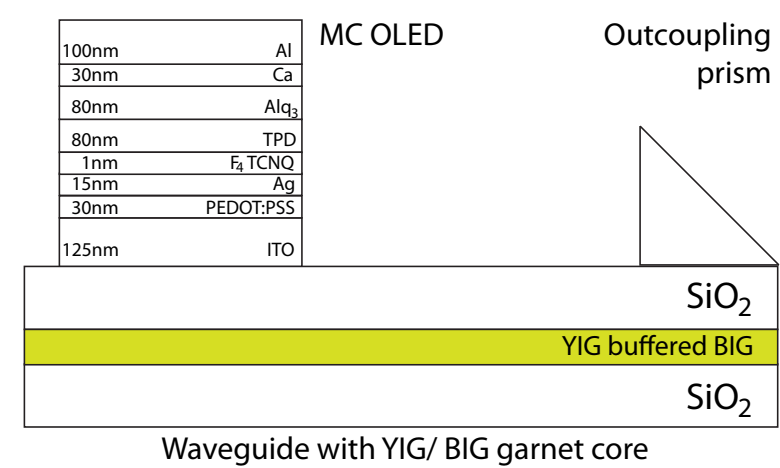

Figure 19 Layout of the modulator setup. The light emitted by the OLED is guided in the garnet containing $\mathrm{SiO}_{2}$ waveguide. A prism is used to outcouple the light from the waveguide into a spectrometer with polarizer.

First the angle dependence of the emission at $532 \mathrm{~nm}$ wavelength was measured. It is shown in Fig. 20a. The difference between maximum and minimum gives the difference in s- and p-polarized light. The measured values are all normalized to the maximum of the s-polarized light. The intensity of p-polarized light is approximately $60 \%$ of the s-polarized light. Second to measure the modulation in the device it was brought into an magnetic field. The field was turned up to $150 \mathrm{mT}$ and $-150 \mathrm{mT}$ and the shown intensity at $532 \mathrm{~nm}$ wavelength was recorded. Figure $20 \mathrm{~b}$ exhibits that the values of the rotation obtained through Faraday-rotation fits well to the data measured by turning the polarizer. This indicates, that a modulation of the light outcouppled from the OLED is possible using the garnet enhanced waveguide.

9 Summary and outlook In this paper it was shown that it is possible to build a hybrid system of OLEDs and a garnet enhanced planar waveguide. A modulation of $1^{\circ}$ per $3.3 \mathrm{mT}$ is possible. The device is encapsulated and can work under normal atmosphere.

To enable this, the garnets properties have been optimized to reduce cracks and roughness in the system. The surface morphology and the substrate influence have been studied. An influence of the substrate on the nucleation is evident, but the garnet growth is only influenced by the 

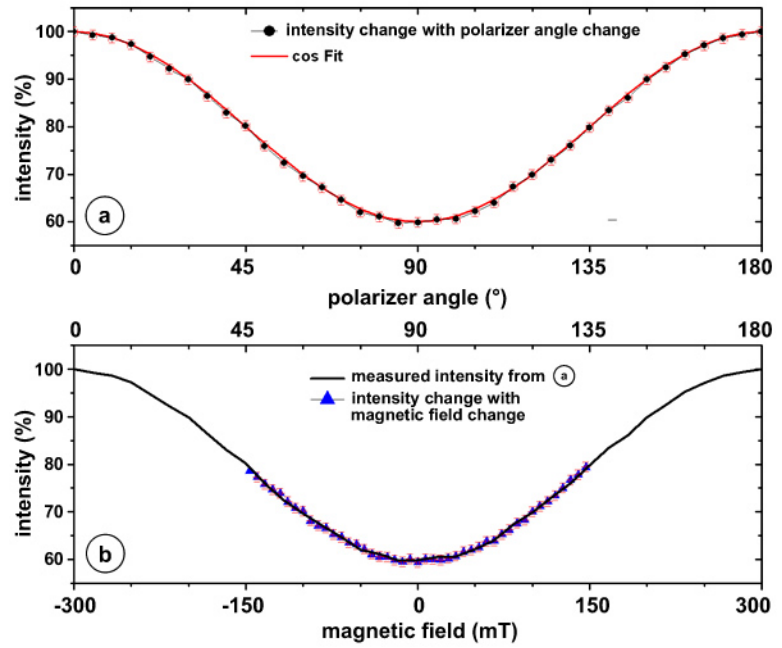

Figure 20 Recorded intensity for a) turning the polarizer b) applying a magnetic field.

properties of the plasma. The reduction of cracks in the surface was possible by choosing the right substrate with an appropriate thermal expansion coefficient. A buffer system based on GGG was developed to study the stress release in the material compared to YIG.

The garnets were structured using laser structuring and ion beam etching. As garnets are comparatively stable against chemical etching these methods seemed feasible. We obtained the best results for laser structured garnet films using a femtosecond laser at a wavelength of 1040 $\mathrm{nm}$ with a pulse duration of $350 \mathrm{fs}$, a pulse energy of 0.8 Watt and a repetition rate of up to $100 \mathrm{kHz}$. Each structuring process was repeated 16 times to optimize the wall roughness.

The optical properties of BIG have been analyzed using ellipsometric measurements. The optical constants could be derived from the obtained data and confirmed by transmissions measurements. The Faraday-rotation of BIG scales linear with the film thickness, thus it is possible to adjust the needed rotation and the BIG thickness for best performance.

To find the best values for the microcavity OLED stack various setups have been simulated. Especially the transition from a normal OLED to a microcavity have been investigated for various materials. From the simulations done regarding the organic layer thickness of microcavity OLEDs only small amount was presented. The optimized parameters for light coupling into the substrate could be found. The simulations were verified by the production of chosen OLED stacks, which showed very could electrical and optical behavior. The diode characteristic of the microcavity OLED was clearly visible and luminance started at $2.5 \mathrm{~V}$ having luminance values comparable to non cavity OLEDs.
In the end we showed the modulation of the microcavity OLEDs light in the garnet enhanced waveguide in response to an external electric field. As the absorption in the waveguide is $20 \mathrm{db} / \mu \mathrm{m}$ at $532 \mathrm{~nm}$ wavelength the thickness of the garnet layer had to be adjusted for best performance. For our model system a $125 \mathrm{~nm}$ thick garnet core was embedded in $\mathrm{SiO}_{2}$.

As BIG shows high absorption in the visible spectral range a shift in the luminance of the microcavity OLED is desirable. This is an interesting prospect for further research in the area. A microcavity OLED with near infrared light emission could solve the problem of absorption and thus lead to an all garnet waveguide.

Acknowledgements The authors would like to thank the Deutsche Forschungsgemeinschaft (Schwerpunktprogramm 1157) for their financial support of this work and S. Zoppel and J. Edlinger at the Research Centre for Microtechnology at the Vorarlberg University of Applied Sciences for the laser structuring.

\section{References}

[1] N.A. Reinke, C. Ackermann, W. Bruetting, Opt. Commun. 266, 191 (2006).

[2] M. Faraday, Phil. Trans. R. Soc. London 136:1, 20 (1846).

[3] L.J. Aplet, J.W. Carson, Appl. Opt. 3, 544 (1964).

[4] H. Doetsch, N. Bahlmann, O. Zhuromskyy, M. Hammer, L. Wilkens, R. Gerhardt, P. Hertel, J. Opt. Soc. 22, 240 (2005).

[5] A. Heinrich, B. Renner, R. Lux, S.G. Ebbinghaus, A. Reller, and B. Stritzker, Thin Solid Films 479, 12 (2005).

[6] T. Koerner, A. Heinrich, M. Weckerle, P. Roocks, B. Stritzker J. Appl. Phys. 103, 07 B337 (2008).

[7] A. Heinrich, T. Koerner, J. Simon, W. Mader, M. Knoll, A. Kalytta, J. Mag. Soc. Jap. 32, 130 (2008).

[8] R. Lux, A. Heinrich, S. Leitenmeier, T. Koerner, M. Herbort, B. Stritzker J. Phys. A 100, 113511 (2006).

[9] G. Winkler, Magnetic Garnets (Vieweg \& Sohn, Braunschweig, 1981).

[10] T. Aichele, A. Lorenz, R. Hergt, and P. Goernert, Cryst. Res. Technol. 38, 275 (2003).

[11] T. Okuda, T. Katayama, H. Kobayashi, N. Kobayashi, K. Satoh, H. Yamamoto, J. Phys. A 367, 4944 (1990).

[12] S. Kahl, S.I. Khartsev, A.M. Grishin, K. Kawano, G. Kong, R.A. Chakalov, J.S. Abell, J. Phys. A 91, 9556 (2002).

[13] S. Kahl, A.M. Grishin, J. Appl. Phys. 94, 5688 (2003).

[14] D. Basting, Excimer Laser Technology: laser sources, optics, systems and applications (Lambda Physik AG, Goettingen, 2001).

[15] D.H.A. Blank, R.P.J. Ijsselsteijn, P.G. Out, H.J.H. Kuiper, J. Flokstra, H. Rogalla, Mater. Sci. Eng. 13, 67 (1992).

[16] D.B. Chrisey, G.K. Hubler, Pulsed laser deposition of thin (Wiley \& Sons, New York, 1994).

[17] A.V. Gusarov, I. Smurov, J. Phys. A 97, 014307 (2005).

[18] R. Kelly, und R.W. Dreyfus, Nucl. Instrum. Meth. 32, 341 (1988).

[19] M. Chern and J. Liaw, Jpn. J. Appl. Phys. 36, 1049 (1997).

[20] M.-B. Park, N.-H. Cho, J. Magn. Magn. Mater. 231, 253 (2001).

[21] B.E. Deal, A.S. Grove, J. Phys. A 36, 3770 (1965). 
[22] H. Kageshima, K. Shiraishi, M. Uematsu Jpn. J. Apll. Phys. 38, L971 (1999).

[23] P.P. Pronko, S.K. Dutta, J. Squier, J.V. Rudd, D. Du, G. Mourou, Opt. Commun. 114, 106 (1995).

[24] C. Momma, B.N. Chichkov, S. Nolte, F. von Alvensleben, A. Tuennermann, H. Welling, Opt. Commun. 129, 134 (1996).

[25] B.N. Chichkov, C. Momma, S. Nolte, F. von Alvensleben, A. Tuennermann, H. Welling, Appl. Phys. A 63, 109 (1996).

[26] B.C. Stuart, M.D. Feit, S. Herman, A.M. Rubenchik, B.W. Shore, M.D. Perry, J. Opt. Soc. 13, 459 (1996).

[27] W. Kautek, J. Krueger, M. Lenzner, S. Sartania, C. Spielmann, and F. Krausz, Appl. Phys. Lett. 69, 3146 (1996).

[28] S. Zoppel, Mikrostrukturierung von Dielektrika und Halbleitern mit ultrakurzen Laserpulsen (FHK Wels, Austria, 2008).

[29] E. Pross, W. Tolksdorf, H. Dammann, Appl. Phys. Lett. 52, 682 (1988).

[30] K.K. Lee, D.R. Lim, H.-C. Luan, A. Agarwal, J. Foresi, L.C. Kimerling, Appl. Phys. Lett. 77, 1617 (2000)

[31] S. Kahl, A.M. Grishin, S.I. Khartsev, K. Kawano, J.S. Abell, IEEE Trans. Magn. 3, 2457 (2001)

[32] K. Kawano, R.A. Chakalov, G. Kong, J.S. Abell, S. Kahl, A.M. Grishin, Physica C, 372376 (2002)

[33] S.H. Wemple, S.L. Blank, J.A. Seman, W.A. Biolsi, Phys. Ref. B, 92134 (1973)

[34] S. Wittekoek, T.J.A. Popma, J.M. Roberston, P.F. Bongers, Phys. Ref. B, 122777 (1975)

[35] C.F. Buhrer, J. Phys. A 40, 4500 (1969).

[36] P. Hansen, K. Witter, W. Tolksdorf, Phys. Rev. B 27, 6608 (1983).

[37] P. Hansen, J.-P. Krumme., Thin Solid Films 114, 69 (1984).

[38] P. Hansen, W. Tolksdorf, K. Witter, IEEE Trans. Magn. 5, 1099 (1984).

[39] N.C. Greenham, R.H. Friend, D.D.C. Bradley, Adv. Mater. 6, 491 (1994)

[40] J.S. Kim, P.K.H. Ho, N.C. Greenham, R.H. Friend, J. Appl. Phys. 88, 1073 (2000).

[41] K. Meerholz, D.C. Mueller, Adv. Funct. Mater. 11, 251 (2001).

[42] N.K. Patel, S. Cina, J.H. Burroughes, IEEE J. Select. Topics Quantum Electron. 8, 346 (2002).

[43] G. Gu et al., Opt. Lett. 22, 396 (1997).

[44] C.F. Madigan, M.H. Lu, J.C. Sturm, Appl. Phys. Lett. 76, $1650(2000)$

[45] T. Yamasaki, K. Sumioka, T. Tsutsui, Appl. Phys. Lett. 76, 1243 (2000).

[46] H.F. Wittmann et al., Adv. Mater. 7, 541 (1995).

[47] N. Takada et al., Appl. Phys. Lett. 63, 2032 (1993).

[48] V. Cimrova, D. Neher, J. Appl. Phys. 79, 3299 (1996).

[49] V. Bulovic et al., Phys. Rev. B 58, 3730 (1998).

[50] T. Nakayama, Y. Itoh, A. Kakuta, Appl. Phys. Lett. 63, 594 (1993).

[51] A. Dodabalapur et al., Appl. Phys. Lett. 64, 2486 (1994).

[52] T. Tsutsui et al., Appl. Phys. Lett. 65, 1868 (1994).

[53] R.R. Chance, A. Prock, R. Silbey, J. Chem. Phys. 60, 2744 (1974).

[54] R.R. Chance, A. Prock, R. Silbey, Adv. Chem. Phys. 37, 1 (1978).

[55] L. Novotny, J. Opt. Soc. Am. A 14, 91 (1997).
[56] W.L. Barnes, J. Mod. Opt. 45, 661 (1998).

[57] J.A.E. Wasey, W.L. Barnes, J. Mod. Opt. 47, 725 (2000).

[58] L.H. Smith, J.A.E. Wasey, I.D.W. Samuel, W.L. Barnes, Adv. Funct. Mater. 15, 1839 (2005).

[59] K. Celebi, T.D. Heidel, M.A. Baldo, Opt. Exp. 15, 1762 (2007).

[60] S. Nowy, J. Frischeisen, W. Bruetting Proc. of SPIE Vol. 7415, 74151C (2009). 